\title{
Medial Prefrontal Cortex Processes Threatening Stimuli in Juvenile Rats
}

\author{
Patricia A Kabitzke*,1,2, Gordon A Barr ${ }^{3}$, Thomas Chan ${ }^{4}$, Harry N Shair ${ }^{1,2}$ and \\ Christoph P Wiedenmayer ${ }^{1,2, \text { स }}$
}

'Department of Psychiatry, Columbia University, New York, NY, USA; ${ }^{2}$ Division of Developmental Neuroscience, New York State Psychiatric Institute, New York, NY, USA; ${ }^{3}$ Department of Anesthesiology and Critical Care Medicine, Children's Hospital of Philadelphia and the University of Pennsylvania School of Medicine, Philadelphia, PA, USA; ${ }^{4}$ Claremont Graduate University, Claremont, CA, USA

\begin{abstract}
To survive, all mammalian species must recognize and respond appropriately to threatening stimuli. In adults, the prelimbic medial prefrontal cortex (mPFC) appears to be involved in fear expression, whereas the infralimbic mPFC mediates fear extinction. In juvenile rats (PN26), the mPFC receives information on potential predators but does not act on it. To test whether the prefrontal cortex is capable of fear regulation in the young organism, we exposed juvenile rats to a threatening or nonthreatening stimulus and assessed fear and brain Fos activation of the mPFC subdivisions, amygdala and periaqueductal gray (PAG). In response to the threat, juveniles froze more, spent more time far from the threat, and had elevated numbers of Fos-positive cells in the prelimbic mPFC, the medial amygdala, and ventral PAG. To test the hypothesis that the mPFC has a dual role in modulating the amygdala and PAG in juveniles, we pharmacologically disinhibited each of the two subdivisions of the MPFC and assessed freezing and downstream activation to the threat. Juvenile rats infused with picrotoxin into the prelimbic mPFC and exposed to a threatening stimulus froze less, spent less time far from the threat, and increased Fos expression. Infusion of picrotoxin into the infralimbic mPFC also reduced fear responding to the threatening stimulus but had no effect on Fos expression. In sum, it appears that the mPFC can process threatening stimuli in juveniles at this age, even though it is normally not involved in the fear responses.

Neuropsychopharmacology (2014) 39, 1924-1932; doi:I0.1038/npp.20I4.40; published online I2 March 20I4
\end{abstract}

\section{INTRODUCTION}

The neural structures that mediate behavioral responses to threat are well characterized in adult rats and include the prefrontal cortex (PFC), amygdala, bed nucleus of the stria terminalis, dorsal premammillary body, and periaqueductal gray of the midbrain (PAG; Bandler and Shipley, 1994; Brandao et al, 1999; Davis, 2000; LeDoux, 2000; Sanders et al, 2003; Davis et al, 2010; Xu et al, 2012). Even at young ages, animals can recognize and respond to threatening situations in an adaptive way (Hofer, 1995; Wiedenmayer and Barr, 1998, 2001b). Yet little is known of the neural substrate of fear regulation early in life.

The medial prefrontal cortex (mPFC) has an important role in emotion regulation (Ongur and Price, 2000; Cardinal et al, 2002; Heidbreder and Groenewegen, 2003), exerting 'top-down' influence on the structures such as the amygdala and PAG that produce behavioral responses

*Correspondence: Dr PA Kabitzke, Department of Psychiatry, Columbia University, I05I Riverside Drive, Unit 40, New York, NY 10032, USA. Tel: +91754| 6979, E-mail: pak2130@columbia.edu Deceased 20 March 2010.

Received I November 2013; revised 26 January 2014; accepted 3 February 2014; accepted article preview online 19 February 2014
(Bush et al, 2000; Miller and Cohen, 2001). The infralimbic $\mathrm{mPFC}$ has inhibitory and the prelimbic $\mathrm{mPFC}$ has excitatory properties in fear regulation (see Sotres-Bayon and Quirk, 2010 for a review). These inhibitory and excitatory properties influence the expression of defensive behaviors through direct projections that modulate outputs of the amygdala and the PAG (LeDoux, 2000; Ohman and Mineka, 2001; Rosen, 2004).

In contrast to the abundance of data in adult rats, the mPFC circuitry that modulates responses to threat is minimally described in young rats. Cells projecting from the $\mathrm{mPFC}$ to the amygdala are rearranged during the first postnatal week (Bouwmeester et al, 2002). By postnatal day (PN)13, neurons in all subdivisions of the mPFC project to the amygdala and the density of projections increases with age (Bouwmeester et al, 2002). In preweaning pups (PN14), the mPFC is not activated by threats (Chan et al, 2011). In adolescent animals (PN38-42), the mPFC is activated and influences freezing (Chan et al, 2011). In contrast, the postweaning juvenile period (PN26) appears to be a critical intermediate time because exposure to a threatening stimulus produces robust freezing behavior and activates the fear circuit yet is unaltered by inactivation of the $\mathrm{mPFC}$ (Chan et al, 2011). Thus, at this age, the MPFC is 'aware' of the threat but does not respond to it as it does in adults. 
The unanswered question is, given that the mPFC is activated by a threat, is its inability to activate the downstream fear circuit due to an immaturity of the $\mathrm{MPFC}$ itself or due to immaturity of its downstream projections and targets?

To investigate whether subdivisions of the $\mathrm{MPFC}$ are capable of modulating behavioral responses to fear stimuli in early life, we tested whether activation of the mPFC can engage fear circuits in response to threat at PN26. To assess this, we disinhibited the mPFC by injection of the $\mathrm{GABA}_{\mathrm{A}}$ receptor antagonist picrotoxin to alter fear behavior and activate the downstream amygdala and PAG targets. If the fear circuit is immature in juveniles, disinhibition of the mPFC would not change fear behavior and the amygdala and PAG would not be active. If, in contrast, the circuitry is in place but not normally activated by the mPFC, disinhibition of the MPFC would intensify the fear response and increase amygdala and PAG activity as measured by higher Fos expression (Hoffman and Lyo, 2002).

\section{MATERIALS AND METHODS}

\section{Animals}

Long-Evans hooded rats were bred under standard laboratory conditions in a colony room with a 12-h light-dark cycle with light onset at 6000 hours. Cages were monitored daily in the morning and evening for the presence of newborn pups and the date of birth was considered as day 0 . Rats were weaned on PN23 and grouped with same-sex littermates in groups of two to four animals. We included only male rats because there are few data on defensive behaviors in females, especially during development. To include them would have required substantial detailing of any differences between males and females, which is outside the scope of these experiments. We considered PN26 animals to be juveniles, corresponding roughly to early preadolescent children. For experiment 1, there were 12-13 juveniles per stimulus (cat or female) for the behavioral analysis. Of these we assayed Fos on six animals per stimulus, due to technical issues unrelated to the data. For experiment 2, there were six to seven subjects per injection site, dose and stimulus for behavioral tests and four to six for the downstream Fos analyses. All tests and treatment procedures were approved by and were in accordance with the Institutional Animal Care and Use Committee of New York State Psychiatric Institute.

\section{Testing Chamber and Behavioral Recordings}

On the day of testing, two groups of three rats each from the same litter were removed from their home cage, weighed and marked for identification with an odor-free marker on their fur. We tested juveniles together to minimize the stress of isolation since the animals were group-housed. Experiments were conducted as detailed previously (Chan et al, 2011). Briefly, they were placed in the testing cage $(46 \times$ $25 \times 21 \mathrm{~cm})$ that was subdivided by a wire-mesh partition positioned in the middle of the cage, forming two equal compartments. The three rats were placed in one compartment that was provisioned with soiled wood shavings that had been taken out of the home cage to simulate the nest area and reduce separation-induced stress. One was an unimplanted pups used in Experiment 1; the other two were implanted with cannulas aimed at the infralimbic or prelimbic aspects of the mPFC (Chan et al, 2011). The data from these latter two subjects are presented in Experiment 2. 'Near' was defined as the half-compartment closest to the wire-mesh partition, 'far' was defined as the half-compartment furthest from the partition. Behavior was recorded first in a 3 -min baseline period followed by a 5 -min period of exposure to a non-threatening or threatening stimulus.

A sexually experienced, unfamiliar and unrelated female was presented as the non-threatening stimulus and a mixed cat fur odor stimulus from a fleece pad was presented as the threatening stimulus (refer to Kabitzke et al, 2011 for cat odor stimulus details). These stimuli are ecologically relevant and age-appropriate. Predators represent a potent threat for young rats and the cat fur odor stimulus was found to be effective in previous studies, eliciting both unlearned and learned contextual fear in juvenile Long-Evans rats (Kabitzke et al, 2011; Kabitzke and Wiedenmayer, 2011). Each subject was exposed to a single stimulus (female or cat fur odor).

The behavior was recorded and video files were scored with Observer XT 7.0 (Noldus Information Technologies, Wageningen, The Netherlands) software by an investigator blind to the treatments of the animals. Behavior categories included 'freezing', which was defined as any posture in which the animal did not exhibit movement except for respiration, time spent in the 'far' side, and the number of transitions between the 'far' and 'near' sections. Freezing and time spent on the far side are expressed as a percentage of the total exposure time; transitions are counts of crossings.

\section{Cannula Implantation and Drug Infusion}

On the afternoon before the testing day (PN25), rats were removed from the home cage, anesthetized by isoflurane inhalation and put in a Kopf stereotaxic apparatus (Kopf Instruments, Tujungan, CA) designed for bilateral cannula implantation. To avoid damage to overlying mPFC subdivisions, cannulas were implanted at an angle with intracranial cannula tips located within the infralimbic and the prelimbic mPFC (Figure 1a). Two holes were drilled and two 23-gauge guide cannulas (Plastics One, Roanoke, VA) were implanted bilaterally into the mPFC: infralimbic mPFC: $2.3 \mathrm{~mm}$ anterior and $2.0 \mathrm{~mm}$ lateral to bregma, lowered $4.2 \mathrm{~mm}$ below the skull at a $16^{\circ}$ angle; prelimbic mPFC: $2.0 \mathrm{~mm}$ anterior and $2.2 \mathrm{~mm}$ lateral to bregma, lowered $3.3 \mathrm{~mm}$ below the skull at a $24^{\circ}$ angle (Chan et al, 2011). The guide cannula was fixed to the skull with dental cement and a dummy cannula was kept in the guide cannula to prevent clogging. After recovering in a holding cage with home cage shavings, the rat was weighed and put back with the littermates.

The next day, the test was carried out. Two cannulated rats and an uncannulated littermate were placed in the testing cage. The cannulated pups were injected with either the drug or vehicle. During infusion, a 30-gauge microinjection cannula was inserted $0.5 \mathrm{~mm}$ beyond the tip of the guide cannula. The microinjection cannula was connected to a $10-\mu$ glass syringe. Drugs were microinjected for $30 \mathrm{~s}$, 

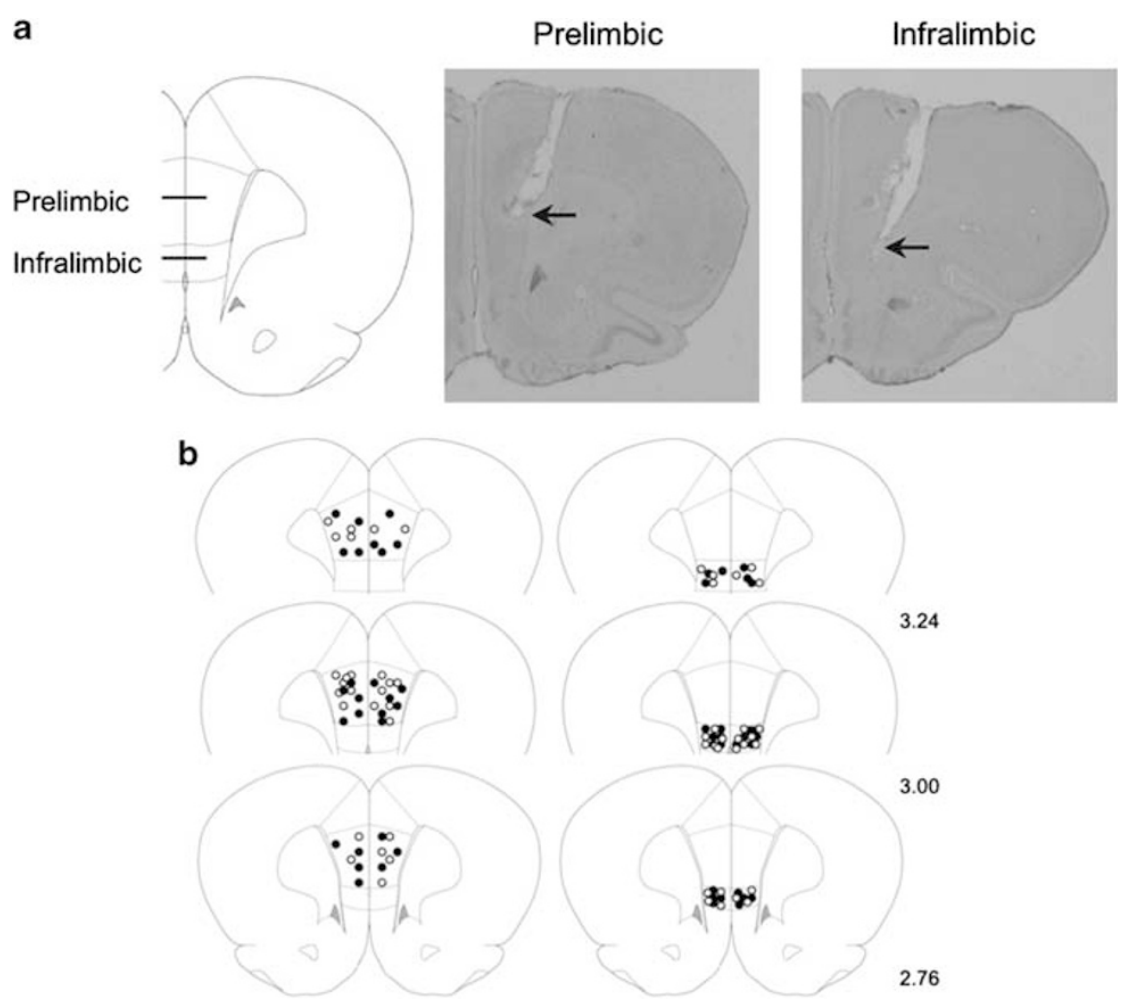

Figure I Cannula placement into the prelimbic and infralimbic mPFC of juvenile rats. (a) Left: A schematic showing the mPFC regions of interest used for the cannula implants and Fos counts in the juvenile rats. Right: Photomicrographs of cresyl-violet-stained sections with placement in prelimbic and infralimbic mPFC. (b) Drawings of placement in prelimbic (left) and infralimbic (right) mPFC. Open circles represent the location of cannula tips in rats infused with vehicle; filled circles represent the location of cannula tips in rats infused with picrotoxin. Numbers indicate the distance in millimeters from bregma.

and the microinjection cannula was left in place for an additional $30 \mathrm{~s}$ to permit diffusion. Ten minutes after infusion, the test continued as described above. After testing, the rats were left undisturbed in a holding cage for $2 \mathrm{~h}$. Rats were given an overdose of a mixture of ketamine $(100 \mathrm{mg} / \mathrm{kg})$ and xylazine $(15 \mathrm{mg} / \mathrm{kg})$, and perfused with $4 \%$ paraformaldehyde. The histological placement of the cannula was determined for each animal by using a brain atlas (Paxinos and Watson, 2007) by an experimenter blind to the treatment groups and to the responses of the rat. Eight animals with cannulas placed outside target areas were excluded from the analysis. Distribution of the injection sites is shown in Figure $1 \mathrm{~b}$.

\section{Drugs}

The $\mathrm{GABA}_{\mathrm{A}}$ receptor antagonist picrotoxin (Sigma, St Louis, MO) was dissolved in isotonic saline, which was also used as the vehicle for control microinjections. Solutions were microinjected in a dose of $300 \mathrm{ng}$ in a volume of $200 \mathrm{nl}$. The dose was determined by pilot studies.

\section{Immunocytochemistry}

The number of Fos-labeled cells was determined by immunocytochemistry $2 \mathrm{~h}$ after testing as described in Chan et al (2011). The brains were frozen and sectioned coronally at $30 \mu \mathrm{m}$ through the $\mathrm{mPFC}$, amygdala, and PAG. Three out of every six sections were collected in $0.1 \mathrm{M}$ phosphate buffer solution (PBS). One section was stained for cresyl violet to locate brain areas and two were used for immunocytochemical processing ( $\mathrm{ABC}$ protocol (Hsu et al, 1981)). Sections of the different conditions were assayed together and incubated for $48 \mathrm{~h}$ at $4{ }^{\circ} \mathrm{C}$ in the primary antibody (rabbit anti-Fos, H-125, Santa Cruz Biotechnology). Stained sections were mounted on gelatin-covered slides, dehydrated in alcohol and xylene, and coverslipped.

\section{Fos Counts and Analysis}

Positively labeled Fos-like cells were visualized using a microscope (Nikon Labophot-2 with $\times 20$ magnification) attached to a digital camera (Nikon DS-Fil) connected to a computer, to identify all Fos-positive cells. Brain nuclei were outlined with the cresyl violet-stained sections using an atlas of the rat brain (Paxinos and Watson, 2007). We counted Fos-like labeled cells in the combined basolateral and lateral nuclei (BLA) and medial nucleus of the amygdala $(\mathrm{MeA})$ and the dorsal (dPAG) and ventrolateral (vPAG) columns of the PAG. All Fos-labeled cells were counted bilaterally in the outlined brain nuclei by an automated counting method using a Macro scripts written for ImageJ (Rasband, 1997-2011). As we were interested in comparing treatments, we counted the profiles of Fos-like IR cells in single optical planes, which is considered appropriate for this type of question (Coggeshall and Lekan, 1996). We collected several sections per brain area and the mean number of cell counts per brain area was calculated by averaging counts from all sections for each animal. Sections of the different conditions and treatments were matched for 

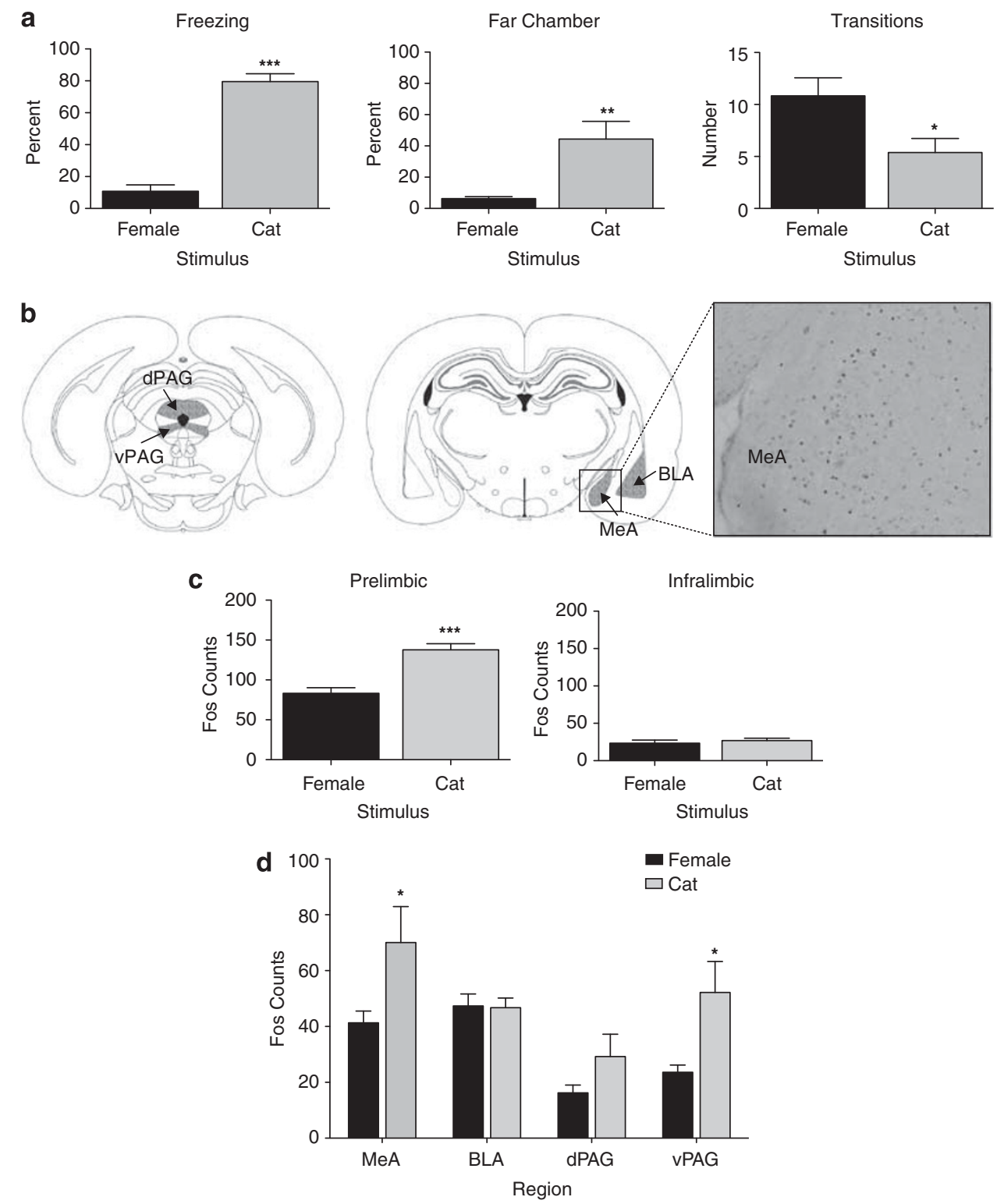

Figure 2 Fear response and Fos expression in juvenile rats with intact mPFC. (a) Percentage time freezing, percentage time in the more distal side of the chamber ('far'), or number of transitions between the near and far side of the chamber during exposure to an adult female rat or cat fur odor (mean \pm SEM). Cat-odor-exposed rats froze significantly more, spent more time farther away, and transitioned between chambers less than did female-exposed rats. $n=12-13$ per stimulus. (b) Schematic of the downstream regions of interest for Fos counts and a representative micrograph to show Fos staining in the MeA of a cat-exposed 26-day-old rat (adapted from Paxinos and Watson, 2007). (c) Activation of the mPFC in intact juvenile rats. Number of Fos-positive cells (mean \pm SE) was significantly increased in prelimbic, but not in infralimbic mPFC, of rats exposed to cat fur odor compared with the adult female rat. (d) Activation of the amygdala and PAG in juvenile rats with intact mPFC. Exposure to cat fur odor significantly increased the number of Fos-positive cells (mean $\pm \mathrm{SE}$ ) in the medial amygdala and ventrolateral PAG. BLA, basolateral/lateral amygdala; MeA, Medial amygdala. $n=6$ in each Fos condition. **** $p<0.001$, *** $p<0.01$, * $p<0.05$.

corresponding neuroanatomical levels. Figure 1a and Figure $2 \mathrm{~b}$ define the regions of interest for the Fos counts.

\section{Statistical Analysis}

For experiment 1, two-way ANOVAs were used to analyze differences between juveniles exposed to the cat fur odor or the female for behavior and Fos expression at each brain site. The two factors were stimulus and brain region. The brain region was a repeated measures factor. Separate
ANOVAs were performed for the mPFC and for downstream structures. Post hoc tests compared the Fos counts for the cat fur odor $v s$ the female for each structure (Sidak multiple comparisons). For experiment 2, there were no differences between the vehicle injection into the two sites and those data were combined for both behavioral and Fos analyses. This resulted in $n=12-14$ for behavioral analysis (freezing, transitions, time spent distant) and for the Fos data, $n=12$ for each amygdala region and $n=7-12$ for each PAG subdivision. Two-way independent ANOVAs were used with the three 
injection conditions as factors (vehicle; picrotoxin into the infralimbic $\mathrm{mPFC}$; picrotoxin into the prelimbic $\mathrm{mPFC}$ ). The dependent variables were freezing, time spent in the far part of the chamber, number of transitions, and Fos counts. Projection areas (amygdala and PAG) were analyzed separately. Post hoc tests compared the response for prelimbic and infralimbic injection to the vehicle condition under the cat and female conditions (Sidak's multiple comparisons). All statistical tests and graphs were generated using GraphPad Prism (v. 6.0 for MacIntosh, San Diego, CA).

\section{RESULTS}

\section{Experiment 1. Response to the Cat Fur Odor}

Behavior. Intact juvenile rats froze significantly more to cat fur odor than to the adult female rat (Figure 2a: $t(23)=10.80, p<0.001)$. They also spent more time in the far side of the testing compartment and transitioned across the compartments less $(t(23)=3.23, p<0.01 ; t(23)=2.50$, $p<0.05$, respectively (Figure $2 \mathrm{a})$ ). This suggests a constellation of behavioral responses to the threat of a cat characterized by less activity/more freezing at a greater distance from the cat fur odor.

Fos staining. Exposure to either of the two stimuli activated the prelimbic subdivision of the MPFC and the cat-odor-exposed rats expressed significantly more Fospositive cells in the prelimbic PFC than did female-exposed rats (significant area $\times$ stimulus interaction: $F(1,10)=35.48$, $p<0.001$ (Figure 2c)). The number of Fos-positive cells did differ between cat- and female-exposed rats in the prelimbic but not in the infralimbic mPFC.

Fos expression differed in the MPFC projection areas as well (Figure 2d). There was a significant Area $\times$ Stimulus interaction $(F(3,30)=6.04, p<0.01))$. Post hoc analysis showed that, compared with female-exposed rats, rats exposed to cat fur odor had significantly more Fos-positive cells than rats exposed to the female in the medial amygdala and vPAG but not the basolateral amygdala or dPAG.

Relationships between freezing and Fos expression in the $m P F C$ and projection sites. To assess this relationship, we correlated Fos expression with freezing. For the mPFC, the prelimbic Fos counts were significantly related to the behavior $(r(10)=0.96)$, whereas the infralimbic mPFC Fos counts were not $(r(10)=0.16)$. This suggests that the infralimbic $\mathrm{mPFC}$ is not receiving relevant input that relates to the perceived threat. In projection areas, Fos and freezing were related and significant in the medial amygdala and vPAG $(r(10)=0.51$ and 0.57 ; one-tailed since we predicted a positive relationship on the basis of the literature and the data in Figure 2) and nonsignificant in the dPAG and basolateral amygdala $(r(10)=0.38$ and 0.10$)$. Scatterplots of these correlations are shown in Figure 3.

\section{Experiment 2. Disinhibition of the mPFC by Picrotoxin Infusion}

To clarify further role of the mPFC in fear regulation in juvenile rats, the infralimbic and prelimbic subdivisions were disinhibited with picrotoxin.
Prestimulus behavior. To test the effects of picrotoxin injection into the mPFC on baseline responding, a 3-min period was assessed immediately before stimulus presentation (empty but novel experimental compartment) in picrotoxin- and vehicle-infused rats (Figure 4a). Picrotoxin reduced freezing and increased transitions compared with vehicle, independent of the site of infusion $((F(1,45)=4.62$, $p<0.05) ; F(1,46)=4.85, p<0.05$, respectively). There were no significant effects on the time spent in the far chamber.

Stimulus-induced behavior. For freezing, there were significant effects of the stimulus $(F(1,45)=65.29$, $p<0.001)$ and treatment $(F(2,45)=4.59, p<0.05)$ but not the interaction (Figure 4b). Post hoc analysis showed a significant difference between vehicle and picrotoxin injected into either the prelimbic or infralimbic PFC for the cat fur odor but not the female $(p<0.001 ; p<0.05$, respectively), suggesting stimulus specificity. Juveniles spent more time far from the cat fur odor but not the female in the vehicle condition and there was no effect of the injection (stimulus effect: $F(1,45)=16.11, p<0.001$ ), showing a fear response to the threat but no suppression by the picrotoxin injection. There were no significant differences in the number of transitions.

Disinhibition of the prelimbic mPFC significantly increased Fos expression in the dPAG (Stimulus $\times$ Injection: $F(2,38)=11.80, p<0.001)$ and medial amygdala (Stimulus $\times$ Injection: $F(2,42)=5.16, p<0.01)$. Post hoc tests showed that prelimbic, but not infralimbic, injection of picrotoxin significantly increased the number of Fos-labeled cells of juvenile rats exposed to cat fur odor in the medial amygdala and dorsal PAG (Figure 4c). There was no effect of mPFC disinhibition on Fos expression the basolateral amygdala or vPAG of rats exposed to cat fur odor. There was also no effect of prelimbic mPFC disinhibition in the amygdala or PAG of rats exposed to the adult female rat.

\section{DISCUSSION}

\section{Summary of Results}

Predator odor is a potent threat to animals in nature and for prey animals; it induces a constellation of behaviors that include freezing, avoiding the threat and/or fleeing. The defense strategy employed depends on the context and the proximity of the threat (Blanchard and Blanchard, 1989; Fanselow, 1994; Kavaliers and Choleris, 2001; Wiedenmayer, 2004). Many laboratory fear procedures rely on a single measure, typically freezing (Blanchard and Blanchard, 1989). The present study employed age-appropriate and ethologically relevant stimuli, and observed other defensive behaviors that are part of a more complicated defensive strategy. The present results confirm and extend findings to include these other behaviors to show that laboratory-reared juvenile rats show unlearned fear to the cat fur odor but not the female (Chan et al, 2011; Kabitzke et al, 2011). This was evidenced by a consistent pattern of behavior that included increased freezing, more time in the distal part of the chamber, and fewer transitions. Thus, the reaction of the juvenile to the threat includes the oftmeasured freezing response, but also other accompanying responses. Fear behavior was accompanied by increased Fos 

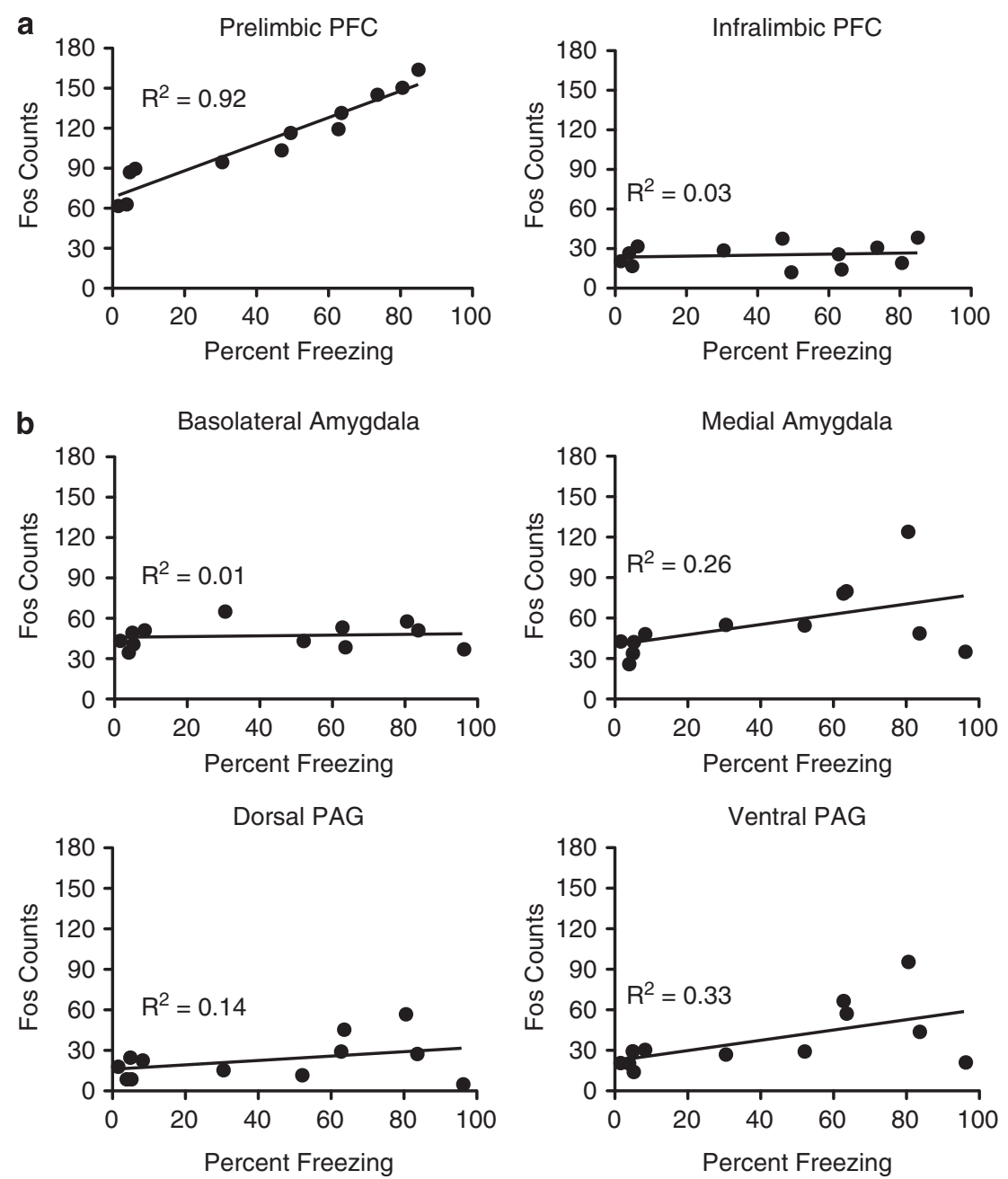

Figure 3 Scatterplots from the correlations between freezing behavior and Fos counts in the mPFC (a) and the downstream amygdala and PAG (b) from experiment I. Each point is a single animal. $n=12$. Note that about half of animals (7 of 12) in (4a) and (4b) are different subjects.

expression in the prelimbic mPFC, medial amygdala, and vPAG. Freezing correlated highly with Fos expression in the prelimbic $\mathrm{mPFC}$, supporting the idea that, to a large extent, prelimbic activation predicts the magnitude of a fear response (Sotres-Bayon and Quirk, 2010). Likewise, downstream structures within the fear circuit, the medial amygdala and vPAG both were activated by the cat fur odor compared with the female and correlated with freezing behavior. The infralimbic mPFC subdivision was not activated by the cat fur odor. In addition, the level of freezing to either stimulus did not correlate to the number of Fos-positive cells in the infralimbic mPFC, suggesting that the infralimbic subdivision of the PFC is not responsive to threat at this age. In adults, the infralimbic mPFC is involved in fear extinction. We know of no data in the immature animal that address the role of infralimbic cortex in fear extinction during development.

Disinhibition of either division of the mPFC with picrotoxin reduced freezing, reduced the time spent in the far chamber, and normalized transitions, disrupting the constellation of fear responses. Picrotoxin injected into the prelimbic $\mathrm{mPFC}$ specifically enhanced Fos expression in the amygdala and dPAG in response to the cat fur odor.
In contrast, in our previous work, inactivation of the mPFC with muscimol did not affect freezing or Fos expression in the amygdala or PAG in infants or in juveniles (Chan et al, 2011). In older adolescent animals, muscimol infused into the prelimbic mPFC decreased freezing and vPAG Fos labeling with cat exposure (Chan et al, 2011).

Consequently, disinhibition by picrotoxin of the prelimbic mPFC had downstream effects in the medial amygdala and vPAG that closely resembled the intact adolescent animal (Chan et al, 2011). It appears that the circuitry that modulates freezing and downstream structures is in place but is not normally activated by the mPFC in juveniles. That freezing would be decreased with disinhibition may indicate a change in defensive strategy from passive to active defense, supported by data that Fos is increased in the dPAG (see discussion below). The mechanisms that allow the developmental transition from quiesence to active function remains to be determined.

\section{Prelimbic mPFC}

The prelimbic mPFC appears poised and capable of regulating fear behavior and the fear circuit but does not 

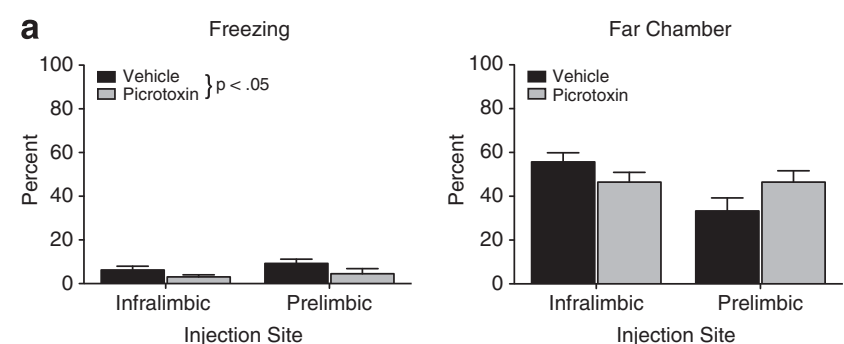

Injection Site
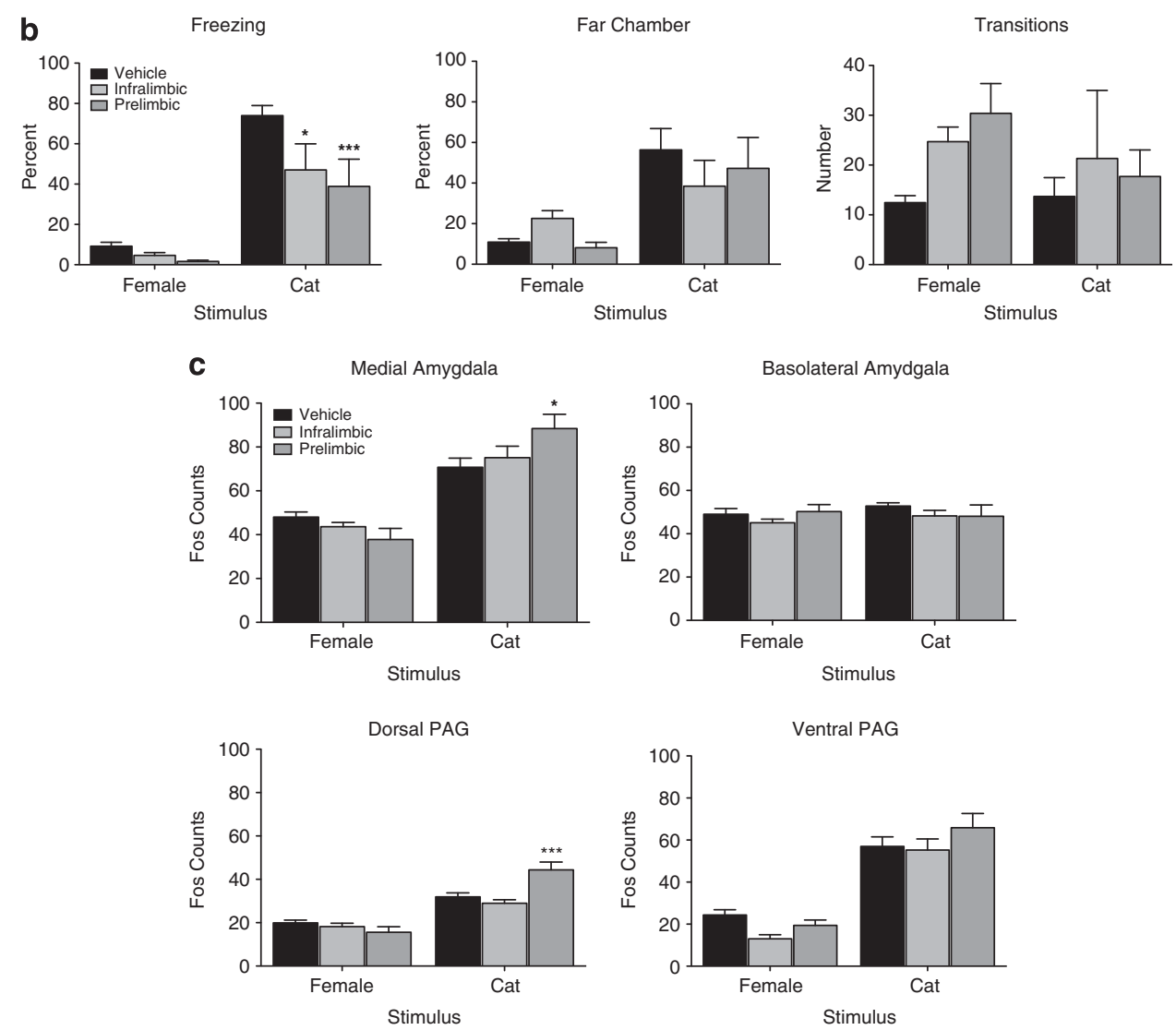

Figure 4 Fear responses and Fos counts following mPFC injections. (a) The behavior of juveniles injected with picrotoxin in the pre-stimulus adaptation period before presentation of the cat fur odor or female. There was increased freezing (left) and more transitions (right) in the picrotoxin-injected group compared with the vehicle-injected group. The site of injection did not differ nor were there effects on the time spent in the distant chamber (center). $n=12-13$ in each injection site. (b) Percentage time freezing, time spent in distal part of the chamber, and number of chamber transitions in juvenile rats infused with picrotoxin or vehicle in $\mathrm{mPFC}$ and exposed to a nonthreatening female or threatening cat fur odor (mean \pm SEM). When infused into the infralimbic or the prelimbic mPFC, freezing to the cat was significantly reduced. $n=6-7$ in each picrotoxin condition, and $n=12-14$ for the vehicle groups. (c) Number of Fos-positive cells in the amygdala and PAG of juvenile rats injected with vehicle or picrotoxin into infralimbic or prelimbic mPFC and exposed to a nonthreatening female or threatening cat fur odor (mean $\pm \mathrm{SE}$ ). Fos expression was significantly increased by picrotoxin compared with vehicle in the $\mathrm{MeA}$ and $\mathrm{dPAG}$ only after prelimbic infusion. There were no effects of picrotoxin in the BLA or the $\mathrm{VPAG}$ on Fos expression. $n=6-7$ in each picrotoxin condition, and $n=8-12$ for the vehicle groups. For all figures, $* * * * * 0.001, * p<0.05$.

yet do so in the juvenile rat. The prelimbic $\mathrm{mPFC}$ receives input that there is a threat: Fos and phosphorylated mitogen-activated protein kinase (pMAPK) expressions are enhanced by cat fur odor in the prelimbic mPFC, the medial amygdala, and ventral PAG (Chan et al, 2011; Li et al, 2012; present data). Furthermore, the levels of freezing significantly correlated with the Fos expression in the prelimbic mPFC. However, inactivation by muscimol of the mPFC did not affect freezing or activation of downstream structures, suggesting that it does not normally participate in freezing behavior at this age (Chan et al, 2011). Activation of the prelimbic mPFC by picrotoxin did reduce freezing and correlated highly with Fos expression in the medial amygdala and the vPAG, suggesting a capability to respond that is not normally engaged. Whether the normal function of the prelimbic mPFC in fear behavior in juveniles is actively inhibited, or whether permissive mechanisms are not yet developed, is not known.

\section{Infralimbic mPFC}

In contrast to the prelimbic mPFC, the infralimbic mPFC does not appear to be involved in the learned or unlearned response to a threat at PN26. Neither Fos (Chan et al, 2011; 
present data) nor pMAPK (Li et al, 2012) increased with exposure to a threat. In addition, there was no significant correlation between freezing and infralimbic Fos expression. Because of these data and data in the adult showing that the infralimbic mPFC mediates fear extinction, we did not expect the reduction in freezing we observed in this picrotoxin-infused group. Interestingly, the infralimbic mPFC appears to be involved in fear extinction at PN23 and in adulthood, but not at PN29 (Pattwell et al, 2012). Thus, the function of the infralimbic mPFC between PN2629 is in transition and remains unclear. However, the lack of correlation of Fos expression in the infralimbic mPFC with any fear behavior suggests that, under normal conditions, the infralimbic mPFC is not engaged in unlearned fear at PN26.

\section{Role of the Amygdala}

The increased Fos expression in the medial but not basolateral amygdala is consistent with data in both weaning age and adult rats. Fos was increased in the basolateral and not the medial amygdala at PN14 in response to a strange male rat, but rapidly switched at PN21 to the adult-like pattern of medial and not basolateral amygdala activation to a threat (Wiedenmayer and Barr, 2001a; Takahashi et al, 2005). Further, freezing is reduced by both with medial ( $\mathrm{Li}$ et al, 2004) and basolateral amygdala activation (Vazdarjanova et al, 2001). These data combined suggest that the basolateral amygdala is not activated by a threat but may have a role in freezing behavior. Of note, the medial but not the basolateral amygdala projects to the bed nucleus of the stria terminalis, a critical structure in the overall fear circuit (Dong et al, 2001). The juvenile amygdala appears to be capable of activation in a manner similar to that in the adult. Fos activation in the medial amygdala, but not the basolateral amygdala, was significantly correlated with freezing. This further suggests that the basolateral amygdala is either not integral to predator-induced fear or functions independently of the remainder of the fear circuit.

\section{Role of the PAG}

In adults, the PAG has been divided anatomically and functionally. The vPAG generates passive (that is, freezing) and the dPAG generates active (that is, jumping) defensive behaviors (Bandler and Shipley, 1994; Fanselow, 1994; Jurgens, 1994; Behbehani, 1995; Davis, 2002). For example, lesions of the ventrolateral column of the PAG abolished unconditioned freezing in various aversive situations such as predator exposure in adult rats (Liebman et al, 1970; De Oca et al, 1998; Farook et al, 2004). The current data suggest that such a division of function exists also in juveniles, as there was a significant increase in Fos expression in the dPAG of cat-exposed, picrotoxin-prelimbic-infused juveniles that was not observed in the vPAG. As the dPAG is thought to be involved in active defense behaviors, this result may signify a developmental change in defensive strategy from passive to active responding (Davis, 2002). However, picrotoxin-infralimbic-infused animals also exhibited a reduction in freezing with no change in $\mathrm{dPAG}$ Fos. Thus, the PAG does not appear to be engaged by mechanisms that reduce freezing with infralimbic activation.

\section{Conclusions}

In sum, freezing behavior in response to threat occurs in rats at all ages; however, neural control differs across ontogeny. Fos expression in the amygdala and PAG across early life demonstrates that these areas are responsive to aversive sensory stimulation and may receive modulatory input from the mPFC. This input seems to convey information about the level of threat and determines that the animal responds appropriately. Although complete understanding of 'upstream' modulation of fear-like responses in early life is still unknown, we have begun to localize where the immaturity of the fear circuit lies. Further study is required to understand how the mPFC influences freezing when disinhibited in juveniles.

\section{FUNDING AND DISCLOSURE}

The authors declare no conflict of interest.

\section{ACKNOWLEDGEMENTS}

When Christoph Wiedenmayer suddenly died in the spring of 2010, a group of his colleagues and students dedicated themselves to completing his research in progress. Although this chapter is now closed, this manuscript by no means represents the end to his work and influence. We gratefully acknowledge Chido Kativhu, Alexei Shemyakin, and Caitlin $\mathrm{McOmish}$ for their research support and Peter Balsam for his thoughtful comments during the preparation of this manuscript. We also thank Michael Myers and the Division of Developmental Neuroscience for continued support. This work is dedicated to the Wiedenmayer family. This work was supported by the National Institutes of Health Grants to CPW: R21 MH073994, R01 MH071751, and ARRA R01 MH071751S1 (HNS and GAB Co-PIs after his death).

\section{REFERENCES}

Bandler R, Shipley MT (1994). Columnar organization in the midbrain periaqueductal gray: modules for emotional expression? Trends Neurosci 17: 379-389.

Behbehani MM (1995). Functional characteristics of the midbrain periaqueductal gray. Progress Neurobiol 46: 575-605.

Blanchard RJ, Blanchard DC (1989). Attack and defense in rodents as ethoexperimental models for the study of emotion. Prog Neuro-Psychopharmacol Biol Psychiatry 13(Suppl): S3-14.

Bouwmeester H, Smits K, Van Ree JM (2002). Neonatal development of projections to the basolateral amygdala from prefrontal and thalamic structures in rat. J Comp Neurol 450: 241-255.

Brandao ML, Anseloni VZ, Pandossio JE, De Araujo JE, Castilho VM (1999). Neurochemical mechanisms of the defensive behavior in the dorsal midbrain. Neurosci Biobehav Rev 23: 863-875.

Bush G, Luu P, Posner MI (2000). Cognitive and emotional influences in anterior cingulate cortex. Trends Cogn Sci 4: 215-222.

Cardinal RN, Parkinson JA, Hall J, Everitt BJ (2002). Emotion and motivation: the role of the amygdala, ventral striatum, and prefrontal cortex. Neurosci Biobehav Rev 26: 321-352. 
Chan T, Kyere K, Davis BR, Shemyakin A, Kabitzke PA, Shair HN et al (2011). The role of the medial prefrontal cortex in innate fear regulation in infants, juveniles, and adolescents. J Neurosci 31: 4991-4999.

Coggeshall RE, Lekan HA (1996). Methods for determining numbers of cells and synapses: a case for more uniform standards of review. JComp Neurol 364: 6-15.

Davis M (2000). The Role of the Amygdala in Conditioned and Unconditioned Fear and Anxiety. Oxford University Press: Oxford.

Davis M (2002). Neural circuitry of anxiety and stress disorders. In: Davis K. L. et al eds Neuropsychopharmacology: The Fifth Generation of Progress. Lippincott Williams \& Wilkins: Philadelphia, PA, USA.

Davis M, Walker DL, Miles L, Grillon C (2010). Phasic vs sustained fear in rats and humans: role of the extended amygdala in fear vs anxiety. Neuropsychopharmacology 35: 105-135.

De Oca BM, DeCola JP, Maren S, Fanselow MS (1998). Distinct regions of the periaqueductal gray are involved in the acquisition and expression of defensive responses. J Neurosci 18: 3426-3432.

Dong HW, Petrovich GD, Swanson LW (2001). Topography of projections from amygdala to bed nuclei of the stria terminalis. Brain Res Brain Res Rev 38: 192-246.

Fanselow MS (1994). Neural organization of the defensive behavior system responsible for fear. Psychonom Bull Rev 1: 429-438.

Farook JM, Wang Q, Moochhala SM, Zhu ZY, Lee L, Wong PT (2004). Distinct regions of periaqueductal gray (PAG) are involved in freezing behavior in hooded PVG rats on the cat-freezing test apparatus. Neurosci Lett 354: 139-142.

Heidbreder CA, Groenewegen HJ (2003). The medial prefrontal cortex in the rat: evidence for a dorso-ventral distinction based upon functional and anatomical characteristics. Neurosci Biobehav Rev 27: 555-579.

Hofer MA (1995). An evolutionary perspective on anxiety. In: Roose SPGlick RA, (eds). Anxiety as Symptom and Signal. The Analytic Press: Hillsdale, pp 17-38.

Hoffman GE, Lyo D (2002). Anatomical markers of activity in neuroendocrine systems: are we all 'fos-ed out'? J Neuroendocrinol 14: 259-268.

Hsu SM, Raine L, Fanger H (1981). Use of avidin-biotin-peroxidase complex $(\mathrm{ABC})$ in immunoperoxidase techniques: a comparison between $\mathrm{ABC}$ and unlabeled antibody (PAP) procedures. J Histochem Cytochem 29: 577-580.

Jurgens U (1994). The role of the periaqueductal grey in vocal behaviour. Behav Brain Res 62: 107-117.

Kabitzke PA, Silva L, Wiedenmayer C (2011). Norepinephrine mediates contextual fear learning and hippocampal pCREB in juvenile rats exposed to predator odor. Neurobiol Learn Memory 96: 166-172.

Kabitzke PA, Wiedenmayer CP (2011). Effects of the stimulus and chamber size on unlearned fear across development. Behav Process 86: 257-262.

Kavaliers M, Choleris E (2001). Antipredator responses and defensive behavior: ecological and ethological approaches for the neurosciences. Neurosci Biobehav Rev 25: 577-586.
LeDoux JE (2000). Emotion circuits in the brain. Ann Rev Neurosci 23: $155-184$.

Li CI, Maglinao TL, Takahashi LK (2004). Medial amygdala modulation of predator odor-induced unconditioned fear in the rat. Behav Neurosci 118: 324-332.

Li S, Kim JH, Richardson R (2012). Differential involvement of the medial prefrontal cortex in the expression of learned fear across development. Behav Neurosci 126: 217-225.

Liebman JM, Mayer DJ, Liebeskind JC (1970). Mesencephalic central gray lesions and fear-motivated behavior in rats. Brain Res 23: 353-370.

Miller EK, Cohen JD (2001). An integrative theory of prefrontal cortex function. Ann Rev Neurosci 24: 167-202.

Ohman A, Mineka S (2001). Fears, phobias, and preparedness: toward an evolved module of fear and fear learning. Psychol Rev 108: 483-522.

Ongur D, Price JL (2000). The organization of networks within the orbital and medial prefrontal cortex of rats, monkeys and humans. Cereb Cortex 10: 206-219.

Pattwell SS, Duhoux S, Hartley CA, Johnson DC, Jing D, Elliott MD et al (2012). Altered fear learning across development in both mouse and human. Proc Natl Acad Sci USA 109: 16318-16323.

Paxinos G, Watson C (2007). The Rat Brain in Stereotaxic Coordinates. Academic: San Diego.

Rasband WS (1997-2011). ImageJ. US National Institutes of Health: Bethesda, Maryland, USA.

Rosen JB (2004). The neurobiology of conditioned and unconditioned fear: a neurobehavioral system analysis of the amygdala. Behav Cogn Neurosci Rev 3: 23-41.

Sanders MJ, Wiltgen BJ, Fanselow MS (2003). The place of the hippocampus in fear conditioning. Eur J Pharmacol 463: 217-223.

Sotres-Bayon F, Quirk GJ (2010). Prefrontal control of fear: more than just extinction. Curr Opin Neurobiol 20: 231-235.

Takahashi LK, Nakashima BR, Hong H, Watanabe K (2005). The smell of danger: a behavioral and neural analysis of predator odor-induced fear. Neurosci Biobehav Rev 29: 1157-1167.

Vazdarjanova A, Cahill L, McGaugh JL (2001). Disrupting basolateral amygdala function impairs unconditioned freezing and avoidance in rats. Eur J Neurosci 14: 709-718.

Wiedenmayer CP (2004). Adaptations or pathologies? Long-term changes in brain and behavior after a single exposure to severe threat. Neurosci Biobehav Rev 28: 1-12.

Wiedenmayer CP, Barr GA (1998). Ontogeny of defensive behavior and analgesia in rat pups exposed to an adult male rat. Physiol Behav 63: 261-269.

Wiedenmayer CP, Barr GA (2001a). Developmental changes in c-fos expression to an age-specific social stressor in infant rats. Behav Brain Res 126: 147-157.

Wiedenmayer CP, Barr GA (2001b). Developmental changes in responsivity to threat are stimulus-specific in rats. Dev Psychobiol 39: 1-7.

Xu HY, Liu YJ, Xu MY, Zhang YH, Zhang JX, Wu YJ (2012). Inactivation of the bed nucleus of the stria terminalis suppresses the innate fear responses of rats induced by the odor of cat urine. Neuroscience 221: 21-27. 